\title{
Controlling the Limit-Cycle of the Ziegler Column via a Tuned Piezoelectric Damper
}

\author{
Francesco D'Annibale, ${ }^{1}$ Giuseppe Rosi, ${ }^{1,2}$ and Angelo Luongo ${ }^{1}$ \\ ${ }^{1}$ International Research Center on Mathematics and Mechanics of Complex Systems, University of L'Aquila, 67100 L'Aquila, Italy \\ ${ }^{2}$ Laboratoire Modélisation et Simulation Multi Echelle, MSME UMR 8208 CNRS, Université Paris-Est, 94010 Créteil, France \\ Correspondence should be addressed to Angelo Luongo; angelo.luongo@univaq.it
}

Received 2 April 2015; Accepted 18 August 2015

Academic Editor: Anna M. Gil-Lafuente

Copyright (c) 2015 Francesco D’Annibale et al. This is an open access article distributed under the Creative Commons Attribution License, which permits unrestricted use, distribution, and reproduction in any medium, provided the original work is properly cited.

This paper is about the nonlinear analysis of a piezoelectric controlled Ziegler column. The piezoelectric controller, here referred to as Tuned Piezoelectric Damper (TPD), possesses evanescent characteristics and, moreover, it is tuned to the first natural frequency of the mechanical system, thus resembling the well-known Tuned Mass Damper. This means that the flow of energy between mechanical and electrical subsystems is driven by the resonance (Den Hartog principle) and magnified by the singularity of the evanescent electrical characteristics. Numerical simulations, showing how the proposed control strategy is effective in increasing the linear stability domain and decreasing the amplitude of the limit-cycles in the postcritical range, are presented.

\section{Introduction}

Structures subjected to follower forces exhibit rich and interesting dynamic features. Among these latter, the most studied are the loss of stability and the onset of several paradoxes (e.g., related to the damping). Moreover, it is interesting to remark that control strategies that are optimal for reducing vibrations can have a detrimental effect on stability (see, e.g., [1]). Indeed, developing a unified control strategy optimized for damping vibrations in the linear and postcritical regimes, and at the same time increasing the stability zone, is a challenging task.

Since their first introduction in the early 1990s, piezoelectric based controllers have attracted the interest of researchers and their industrial applications have increased over the past years. These controllers are usually divided into two main categories: active and passive controllers. The distinction is based on whether or not the controller is able to pump energy into the mechanical system. As it is well known, active controllers can be really effective but also suffer from stability issues. Examples of active controllers can be found in $[2,3]$. This is why our choice is to focus our attention on passive controllers of the type firstly introduced in [3]. As they are usually implemented by tuning the resonance(s) of the electric circuit to one (or more) natural frequency(ies) of the mechanical system, they can represent a valid alternative to the classic Tuned Mass Dampers (TMDs) [4-8]. Their advantage is mostly due to the fact that they are more versatile, simpler to tune, and in most configurations lighter than classic TMD. Moreover, piezoelectric controllers can be used also in microdevices [9].

In our previous studies [10,11], we focused our attention on several kinds of piezoelectric control strategies, applied to discrete linear structures. In these papers, mechanical and electrical subsystems were studied in their linear and nonlinear dynamics, respectively. In [10], three different controllers were proposed and it was proved that, at various extents, they all have a beneficial effect on stability. In [11], attention was focused on a two-DOF system, namely, the nonlinear Ziegler column, which was taken as case study, and the amplitude of the limit-cycle was investigated with and without the piezoelectric controller: in particular, in 


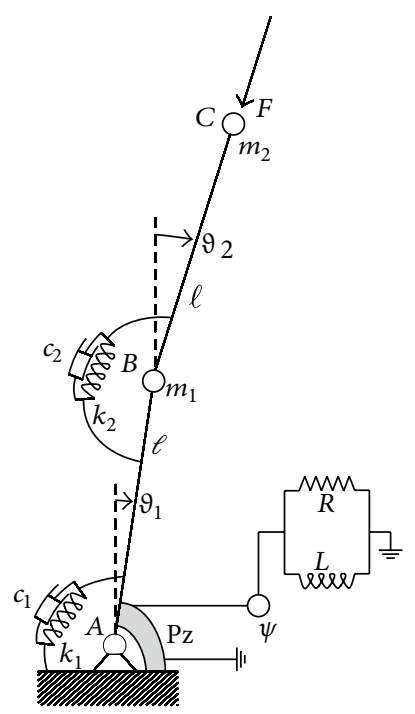

FIgURE 1: Nonlinear controlled Ziegler column.

that paper we used one of the three controllers defined in [10], namely, the SNRC (Singular Nonresonant Controller), which remembers the Nonlinear Energy Sink (see, e.g., [12, 13]) even if, differently from the NES, the coupling between the mechanical system and the controller is linear and of gyroscopic type.

The objective of this paper is to study the behavior of the nonlinear Ziegler column equipped with another one of the three controllers introduced in [10], which is called the SRC (Singular Resonant Controller), which resembles the working mechanism of the TMD, since it is resonant and possesses evanescent electric properties. This type of controller, therefore, can be also referred to as Tuned Piezoelectric Damper (TPD). It is important to remark that we include into the category of TPD only passive circuits, that is, circuits that cannot pump energy into the mechanical systems. Few examples of controllers having these characteristics can be found in $[14,15]$ for collocated control and in [16-24] for distributed control; moreover, if the circuit parameters are well optimized, good damping results can be obtained even without resonant elements (see, e.g., [25]).

The paper is structured as follows. In Section 2, the discrete model of a piezoelectric controlled nonlinear Ziegler column is recalled. Section 3 is devoted to the presentation of the numerical results. Finally, in Section 4 some conclusions and a summary of the main results are exposed.

\section{The Model}

The system under study is a piezoelectric controlled upward double-pendulum, shown in Figure 1. The equations of motion for this system are derived in [11] for finite rotations and will be here briefly recalled. The system consists of a two hinged weightless rigid bars of equal length $\ell$, carrying two concentrated masses, $m_{1}:=2 m$ at the common hinge (point $B$ in the figure) and $m_{2}:=m$ at the tip (point $C$ in the figure). The bars are viscoelastically constrained at the hinges by (a) linear springs of stiffness $k_{1}:=k$ and $k_{2}:=k$ and (b) linear dashpots having viscosity coefficients $c_{1}$ and $c_{2}$, respectively. The system is loaded at the free end by a follower force of intensity $F$, which keeps its direction parallel to the upper bar. Moreover, the pendulum is equipped with a piezoelectric device of stiffness $k_{p}$ (here considered negligible with respect to the stiffness of the springs), capacitance $C_{p}$, and coupling coefficient $g$, which is placed at the ground hinge, and it is connected to a one-node LR resonant circuit, of inductance $L$ and resistance $R$ (sketched in Figure 1), and to the ground. When considering infinitesimal rotations of the two bars, the system reduces (except for the masses values adopted here) to that analyzed in [10]; moreover, when the controller is removed, the system degenerates into the well-known Ziegler column [26].

The state of the system is described by taking as Lagrangian coordinates the rotations of the two bars, $\vartheta_{1}(t)$ and $\vartheta_{2}(t)$, and the flux linkage $\psi(t)$ associated with the piezoelectric actuator. We recall here that the flux linkage is defined as the time integral of the voltage $V$; that is, $\psi=$ $\int V d t$.

The equations of motion for the coupled system are obtained by following these steps:

(1) The internal dissipation (due to damping) and nonconservative action (due to the follower force) are neglected, so that we can describe the system in terms of its Lagrangian, which is the sum of three components: (a) the mechanical Lagrangian $L_{m}$, concerning the structure without any control devices, (b) the electrical Lagrangian $L_{e}$, relevant to the electrical circuit, and (c) the piezoelectric Lagrangian $L_{p}$, referred to as the piezoelectric device.

(2) The internal dissipation and the nonconservative action are accounted for through the Extended Hamilton Principle.

For more details about this process, the reader can refer to $[10,11]$.

The obtained nondimensional system of equations has the following form:

$$
\begin{aligned}
& 3 \ddot{\vartheta}_{1}+\ddot{\vartheta}_{2} \cos \left(\vartheta_{1}-\vartheta_{2}\right)+\dot{\vartheta}_{2}^{2} \sin \left(\vartheta_{1}-\vartheta_{2}\right)+\left(\xi_{1}+\xi_{2}\right) \dot{\vartheta}_{1} \\
& \quad-\xi_{2} \dot{\vartheta}_{2}+2 \vartheta_{1}-\vartheta_{2}-\mu \sin \left(\vartheta_{1}-\vartheta_{2}\right)-\gamma \dot{\psi}=0, \\
& \ddot{\vartheta}_{2}+\ddot{\vartheta}_{1} \cos \left(\vartheta_{1}-\vartheta_{2}\right)-\dot{\vartheta}_{1}^{2} \sin \left(\vartheta_{1}-\vartheta_{2}\right)+\xi_{2}\left(\dot{\vartheta}_{2}-\dot{\vartheta}_{1}\right) \\
& \quad+\left(\vartheta_{2}-\vartheta_{1}\right)=0, \\
& \nu_{e} \ddot{\psi}+\xi_{e} \dot{\psi}+\kappa_{e} \psi+\gamma \dot{\vartheta}_{1}=0,
\end{aligned}
$$


where the superposed dot denotes a time derivative and where we introduced the following quantities (accounting for $m_{1}=2 m, m_{2}=m$, and $k_{1}=k_{2}=k$ ) for nondimensionalization:

$$
\begin{aligned}
\tau & =\omega t, \\
\omega^{2} & =\frac{k}{m \ell^{2}}, \\
\mu & =\frac{F}{m \ell \omega^{2}}, \\
\xi_{1} & =\frac{c_{1}}{m \ell^{2} \omega}, \\
\xi_{2} & =\frac{c_{2}}{m \ell^{2} \omega}, \\
\widetilde{\psi} & =\frac{\psi}{\psi_{0}}, \\
\nu_{e} & =\frac{C_{p}}{C_{0}}, \\
\psi_{0} & =\ell \frac{\sqrt{\frac{m}{C_{0}}},}{\omega \ell \sqrt{m C_{0}}}, \\
\xi_{e} & =\frac{1}{R C_{0} \omega}, \\
\kappa_{e} & =\frac{1}{L C_{0} \omega^{2}},
\end{aligned}
$$

in which $C_{0}$ and $\psi_{0}$ are scaling capacitance and flux linkage, respectively. Here, $0<\mu \in \mathbb{R}$ is the load parameter (taken as bifurcation parameter); $0<\gamma \in \mathbb{R}$ is the electromechanical coupling parameter; $\nu_{e}, \xi_{e}$, and $\kappa_{e}$ (all positive and reals) are electrical parameters, here referred to as the "electrical mass," "electrical damping," and "electrical stiffness," respectively.

\section{Numerical Results}

Numerical results, relevant to the uncontrolled and controlled Ziegler column, in both linear and nonlinear regimes are discussed in this section. It should be remarked that all the bifurcation diagrams discussed in the following have been obtained via a numerical continuation procedure, which has been directly applied to system (1), in order to compute the amplitudes of the limit-cycles occurring in the postcritical regime.

3.1. Uncontrolled Column. This section is devoted to briefly resume the critical and postcritical behavior of the uncontrolled Ziegler column with the aim of preparing the discussion on the effects of the controller, given in the next section.

The linear stability domain of the uncontrolled column, that is, when $\gamma=0$, can be exactly determined by making use of the Routh-Hurwitz criterion on the (complete degree-4) characteristic equation of the algebraic eigenvalue problem associated with the linearized equations (1) (see, e.g., [27]). The criterion leads to the finding of a critical locus in the $\left(\mu, \xi_{1}, \xi_{2}\right)$-space, known in the literature as the "Whitney's umbrella" surface $[28,29]$, whose equation reads as follows:

$$
\mu=\mu_{c}+\frac{\xi_{1} \xi_{2}}{2}-\frac{(3-2 \sqrt{2})}{2} \frac{\left(\xi_{1}-(4+5 \sqrt{2}) \xi_{2}\right)^{2}}{\left(\xi_{1}+\xi_{2}\right)\left(\xi_{1}+6 \xi_{2}\right)},
$$

where $\mu_{c}:=7 / 2-\sqrt{2} \simeq 2.09$ is the critical load of the undamped system at which a circulatory Hopf bifurcation occurs.

The linear bifurcation diagram of the uncontrolled Ziegler column is displayed in Figure 2. In particular, Figure 2(a) shows the critical surface, given by (3), in the 3D domain $\left(\mu, \xi_{1}, \xi_{2}\right)$; it separates the stable states (the region marked with $S$ in the figure) from the unstable ones (the region marked with $U$ in the figure). The points on the surface represent the Hopf bifurcation states, that is, except for those on the $\mu$-axis that, indeed, are regarded as marginally stable (undamped) systems for which a circulatory Hopf bifurcation occurs at $\mu=\mu_{c}$ (see, e.g., [30]). In Figure 2(b), the contour lines $\mu=$ const are displayed: it is apparent that the effect of damping is mostly detrimental in the whole $\left(\xi_{1}, \xi_{2}\right)$-plane, since, except for a small region (filled in gray in the figure) close to an "optimal direction" (dashed line in the figure), the critical load of the damped system $\mu_{d}$ is lower with respect to $\mu_{c}$. Moreover, it can be shown that on the left side of the optimal direction the stability is governed by the first mode, while on the right side it is governed by the second one.

The postcritical behavior of the uncontrolled Ziegler column is now discussed. In particular, we will refer to two systems, marked with a black dot and a label I or II in Figure 2(b), respectively, far and close enough to the critical load of the circulatory system, namely,

(i) case study I: $\xi_{1}=0.05$ and $\xi_{2}=0.2$, entailing $\mu_{d} \simeq$ 0.80 , for which damping has a strong destabilizing effect $(-62 \%)$;

(ii) case study II: $\xi_{1}=0.3$ and $\xi_{2}=0.15$, entailing $\mu_{d} \simeq$ 1.81 , for which damping has a moderate destabilizing effect $(-13 \%)$.

In Figures 3 and 4, the bifurcation diagrams of the uncontrolled Ziegler column are represented for the case studies I and II, respectively. Here, the amplitudes of the motion components, $\max \left|\vartheta_{1}\right|$ and $\max \left|\vartheta_{2}\right|$, are plotted versus the bifurcation parameter $\mu$. It is apparent that also when the bifurcation parameter slightly exceeds its critical value $\mu_{d}$, 


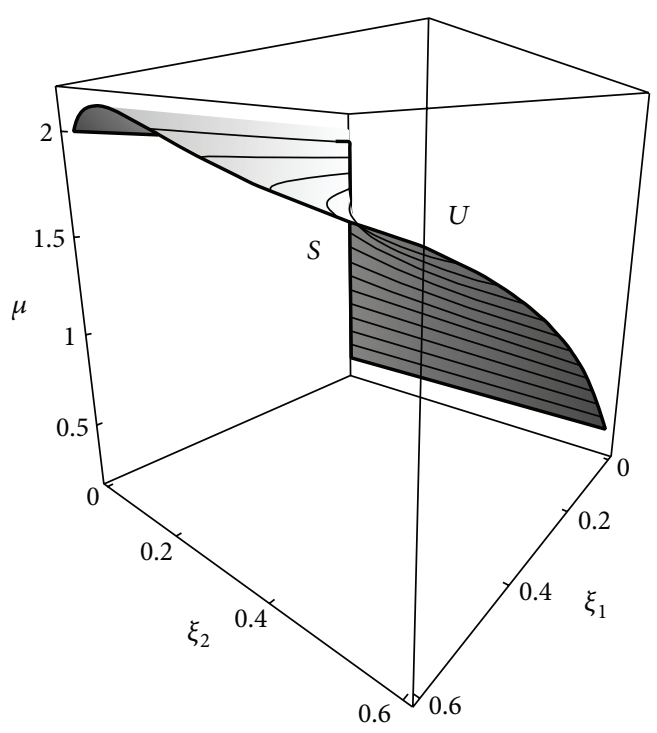

(a)

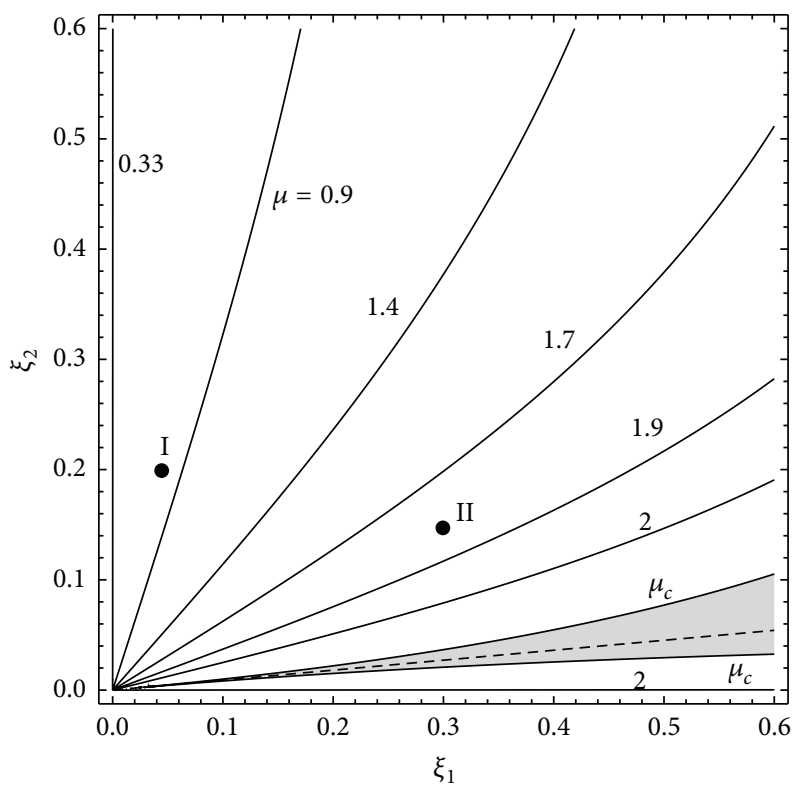

(b)

FiguRE 2: Uncontrolled Ziegler column: (a) critical manifold in the $\left(\mu, \xi_{1}, \xi_{2}\right)$-parameter space: $S$ is stable region and $U$ is unstable region; (b) $\mu$-isolines.

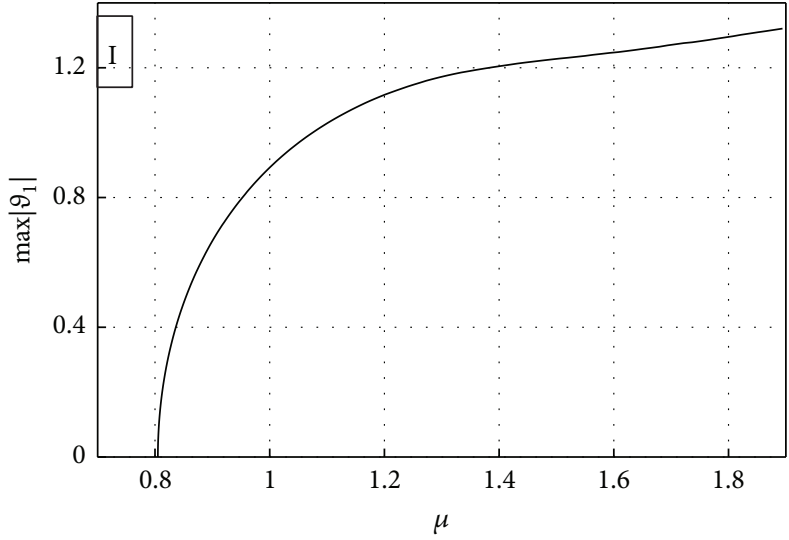

(a)

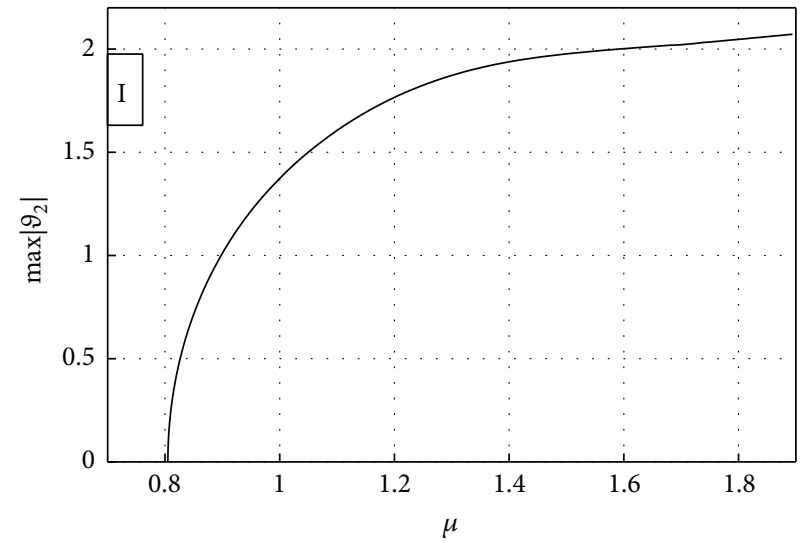

(b)

FiguRE 3: Bifurcation diagrams of the uncontrolled Ziegler column for the case study I: (a) $\max \left|\vartheta_{1}\right|$ versus $\mu$; (b) $\max \left|\vartheta_{2}\right|$ versus $\mu$.

large amplitudes limit-cycles manifest themselves. This is the consequence of the destabilizing effect of damping yet discussed with regard to the linear behavior of the column, which persists also in the postcritical regime. As a matter of fact, when the increment of the load with respect to the critical value, $\delta \mu:=\mu-\mu_{d}$, is equal, for example, to $\delta \mu=0.3$, we found $\max \left|\vartheta_{1}\right| \simeq 1.03 \mathrm{rad}, \max \left|\vartheta_{2}\right| \simeq 1.62 \mathrm{rad}$ in case $\mathrm{I}$ and $\max \left|\vartheta_{1}\right| \simeq 0.52 \mathrm{rad}, \max \left|\vartheta_{2}\right| \simeq 1.13 \mathrm{rad}$. Therefore, the higher the destabilizing effect of damping on linear stability, the higher the amplitude of the limit-cycle (for the same increment of load with respect the critical one).
3.2. Controlled Column. In this section, we analyze the behavior of the Ziegler column equipped with the SRC (Singular Resonant Controller) issued from the paper [10]. As it was proved there through a perturbation technique, the energy flow between mechanical and electrical subsystems is driven, in this controller, by the resonance (Den Hartog principle [31]) and magnified by the singularity of the evanescent electrical characteristics. Thus, according to this control strategy we need to have $\kappa_{e}=\mathscr{O}(\varepsilon), v_{e}=\mathscr{O}(\varepsilon), \xi_{e}=\mathcal{O}\left(\varepsilon^{3 / 2}\right)$, and $\gamma=\mathcal{O}(\varepsilon), \varepsilon$ being a small parameter; moreover, in order to render the electrical oscillator resonant to the mechanical 


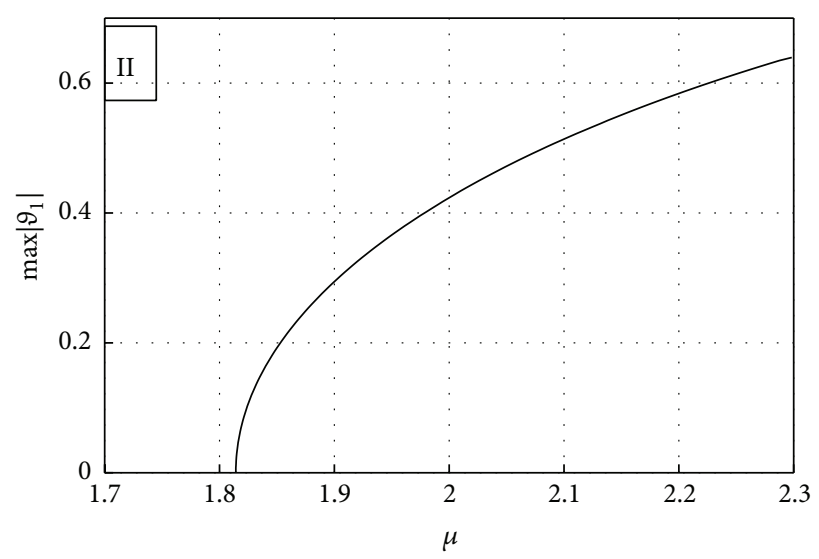

(a)

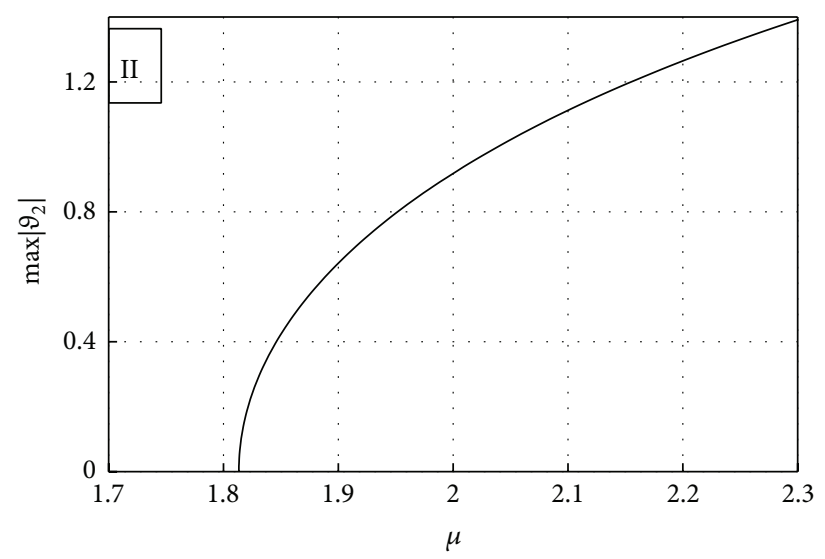

(b)

FIGURE 4: Bifurcation diagrams of the uncontrolled Ziegler column for the case study II: (a) $\max \left|\vartheta_{1}\right|$ versus $\mu$; (b) max $\left|\vartheta_{2}\right|$ versus $\mu$.

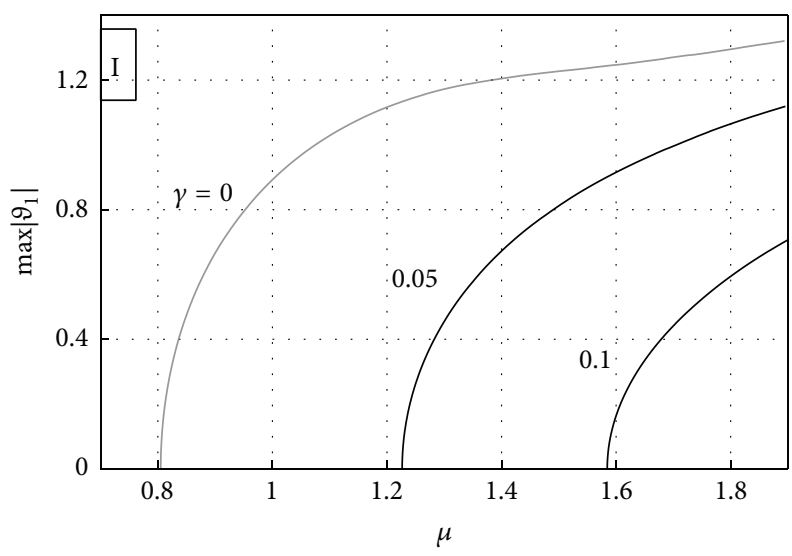

(a)

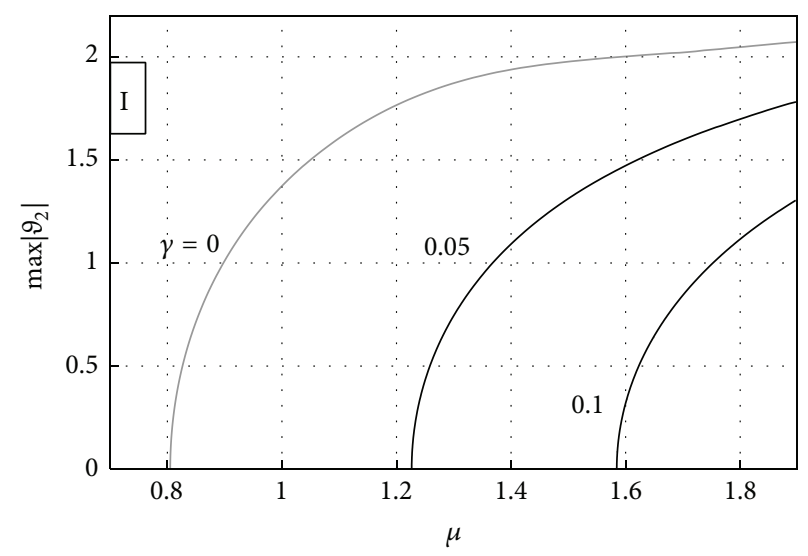

(b)

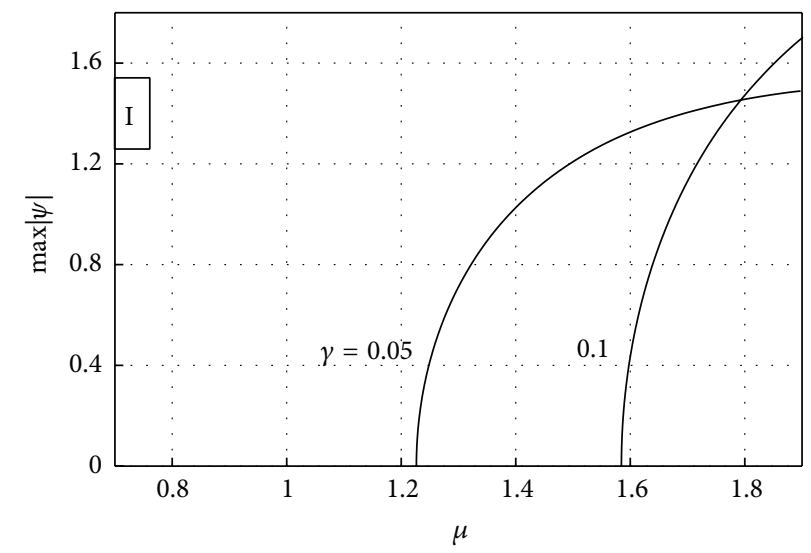

(c)

Figure 5: Bifurcation diagrams of the controlled Ziegler column for the case study I: (a) $\max \left|\vartheta_{1}\right|$ versus $\mu$; (b) max $\left|\vartheta_{2}\right|$ versus $\mu$; (c) max $|\psi|$ versus $\mu$. 


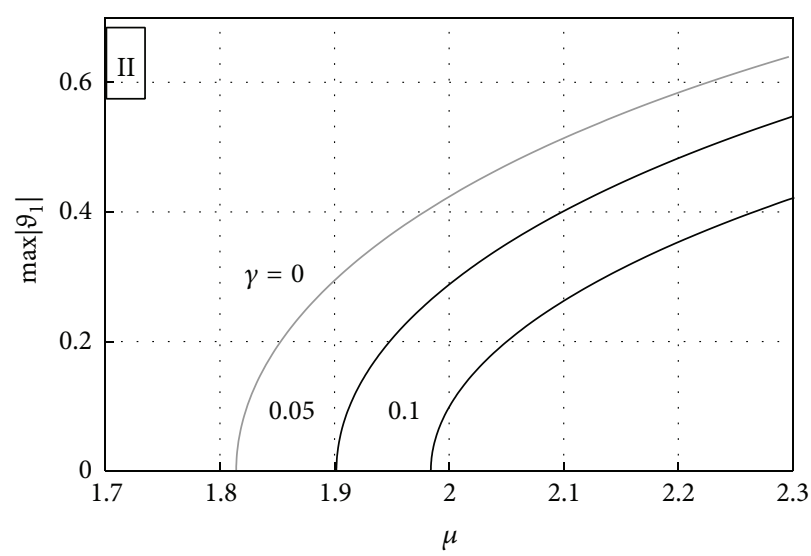

(a)

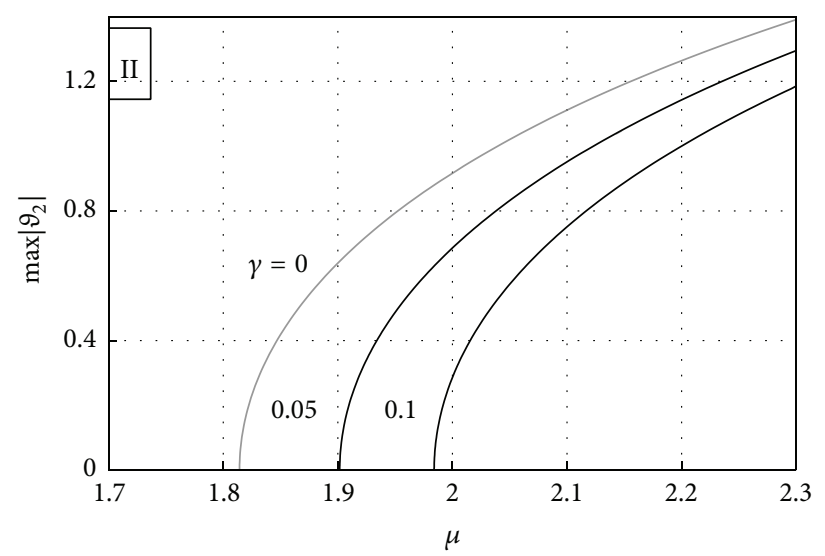

(b)

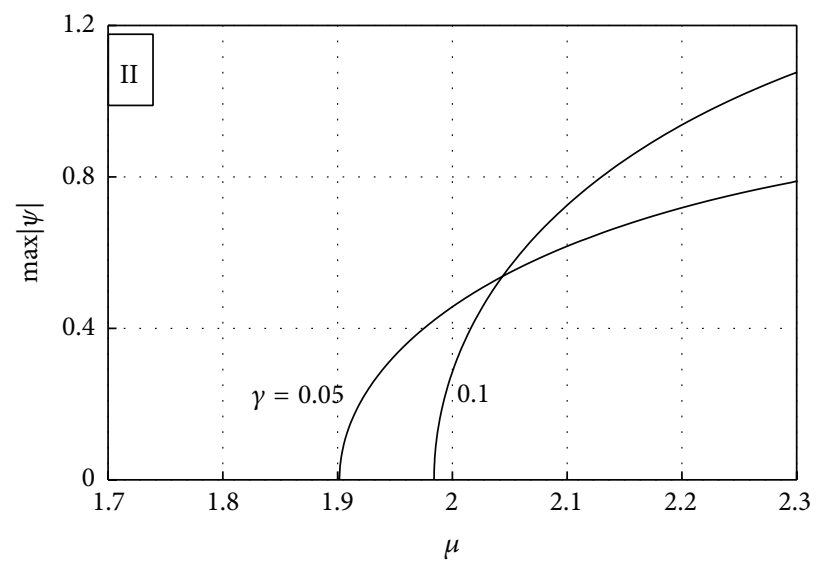

(c)

Figure 6: Bifurcation diagrams of the controlled Ziegler column for the case study II: (a) $\max \left|\vartheta_{1}\right| \operatorname{versus} \mu$; (b) $\max \left|\vartheta_{2}\right|$ versus $\mu$; (c) max $|\psi|$ versus $\mu$.

one, the tuning $\kappa_{e} / \nu_{e}=\omega_{d}^{2}$, where $\omega_{d}$ is one of the two column's frequencies at $\mu=\mu_{d}$, must be performed. It is important to remark that such a controller resembles the wellknown Tuned Mass Damper [4-8] even if the coupling is here linear and is of gyroscopic type; thus, the controller discussed in the present paper can be regarded as a Tuned Piezoelectric Damper (TPD).

According to this strategy, the following electrical parameters have been fixed: $\nu_{e}=0.1$ and $\xi_{e}=0.03$; moreover, $\kappa_{e}$ has been chosen in order to tune the electrical frequency to the first mechanical one since, in both case studies, the first mode is the candidate to become unstable, namely, $\kappa_{e}=0.02$ $\left(\omega_{d} \simeq 0.447\right)$ for case study I and $\kappa_{e}=0.0375\left(\omega_{d} \simeq 0.612\right)$ for case study II. The coupling parameter $\gamma$ has been considered variable, with the aim of estimating the sensitivity of the controller's performances with respect to an increase of the electromechanical coupling and, in particular, it has been fixed to $\gamma=0.05$ or $\gamma=0.1$.

Concerning the linear stability of the controlled system, the effects of the controller on the critical load can be evaluated in Figures 5 and 6 where the bifurcation diagrams of the controlled (black curves) and uncontrolled (gray curves) Ziegler column, for cases I and II, respectively, are represented. It is apparent that, in both case studies, the controller is able to shift forward the uncontrolled bifurcation diagrams, thus entailing an increase of the stability region; in particular, we found the following in the two cases: (I) $\mu_{d} \simeq 1.23(+54 \%)$, when $\gamma=0.05$, and $\mu_{d} \simeq 1.58(+98 \%)$, when $\gamma=0.1$; (II) $\mu_{d} \simeq 1.90(+5 \%)$, when $\gamma=0.05$, and $\mu_{d} \simeq 1.98(+9 \%)$, when $\gamma=0.1$. Therefore, the controller is much more efficient in case study I where, remarkably, the damping has a strong destabilizing effect.

Figures 5 and 6 illustrate also the influence of control on the postcritical behavior of the column: indeed, the TPD is able to slightly flatten the curves on the $\mu$-axis, at least in a range of load values, thus reducing the limit-cycles amplitude of the uncontrolled system. As a matter of fact, we found, when $\delta \mu=0.3$, for the case study I $\max \left|\vartheta_{1}\right| \simeq 0.84 \mathrm{rad}$ $(-18 \%), \max \left|\vartheta_{2}\right| \simeq 1.36 \mathrm{rad}(-16 \%)$, when $\gamma=0.05$, and $\max \left|\vartheta_{1}\right| \simeq 0.69 \operatorname{rad}(-33 \%), \max \left|\vartheta_{2}\right| \simeq 1.28 \mathrm{rad}(-21 \%)$, when $\gamma=0.1$.

It is important to remark that, as a result of the shift, the controller reduces the amplitude of the limit-cycle occurring at the same level of load. This effect is displayed in Figure 7 

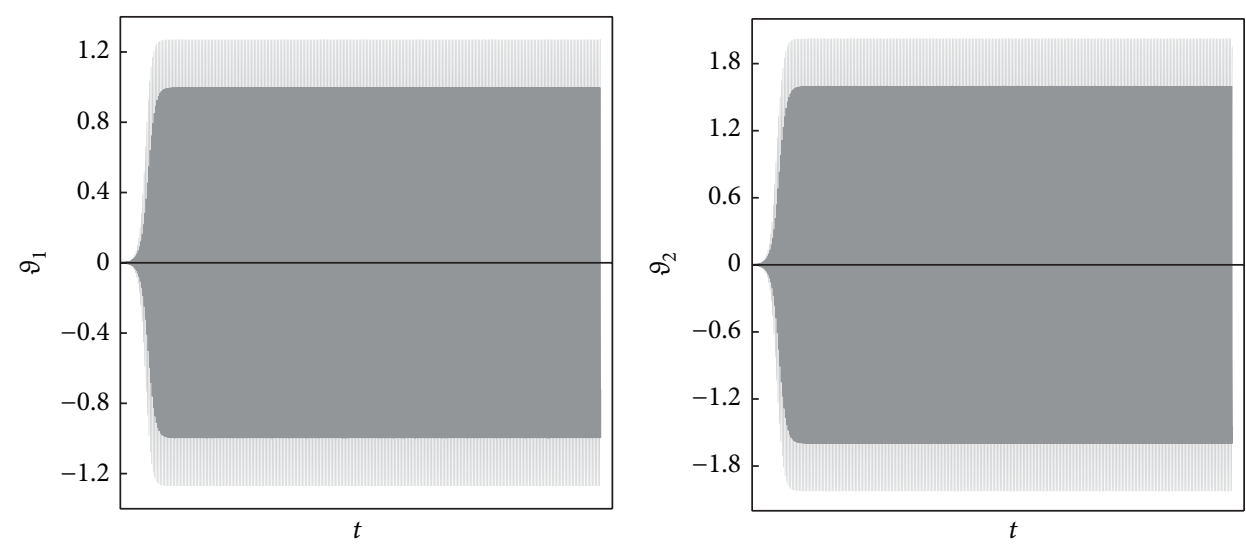

(a)
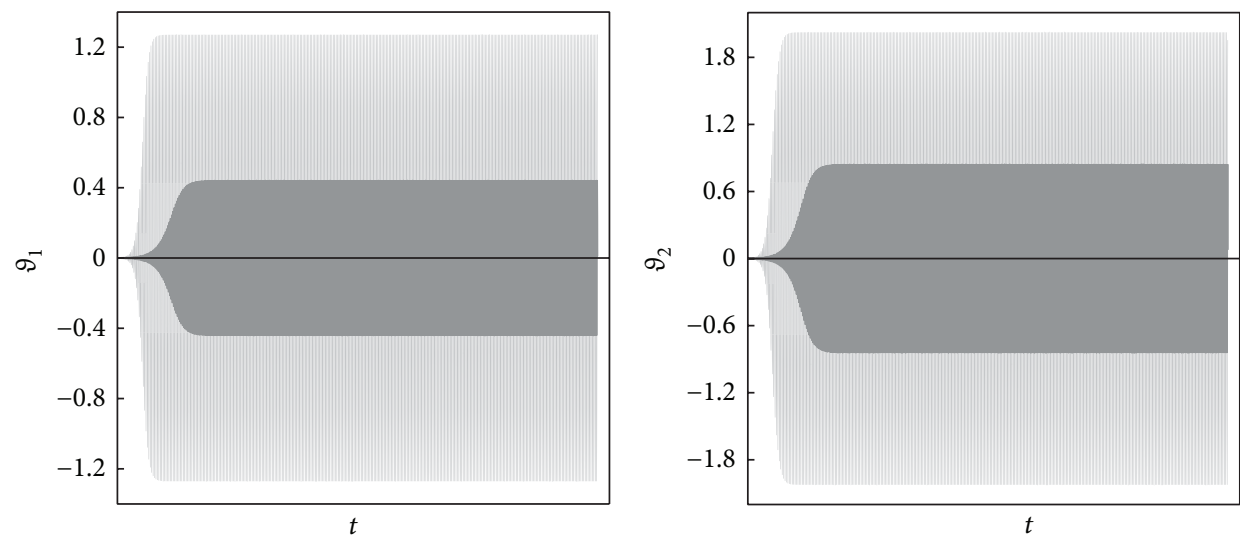

(b)

FIGURE 7: Time histories of $\vartheta_{1}$ and $\vartheta_{2}$ for the case study I, when $\mu=1.7$, for the uncontrolled (light gray curves) and the controlled (dark gray curves) systems when (a) $\gamma=0.05$ and (b) $\gamma=0.1$.

where, for case study I, when $\mu=1.7$, and for the two considered values of $\gamma$, the time histories, relevant to uncontrolled (light gray curves in the figure) and controlled (dark gray curves in the figure) systems, show the benefits of the control. Finally, the flattening of the curves can be seen in Figure 8 where numerical integrations relevant to the uncontrolled system (first column in the figure) and the controlled system (second column in the figure), obtained for two different values of $\mu$, at the same distance $\delta \mu$ from the respective critical loads, are displayed, for case study I, when $\delta \mu=0.3$ and $\gamma=0.1$. In Figures 8(a), 8(b), and 8(c) the projections of the trajectories onto phase planes are plotted, showing the contraction of the amplitude of the limit-cycle.

\section{Conclusions}

In this paper, the effects of a TPD controller on the critical and postcritical behavior of the Ziegler column have been addressed.

The nonlinear equations of motion, for the coupled PiezoElectro-Mechanical (PEM) system, have been recalled. The adopted control strategy first introduced in [10] is the SRC, namely, the Singular Resonant Controller, and its effects on the nonlinear dynamics of the system have been deeply investigated.

The main results can be summarized in the following points:

(i) The analysis of the postcritical scenario shows that the controller is able to shift forward the bifurcation diagrams of the uncontrolled system.

(ii) The controller induces a contraction of the limit-cycle amplitudes.

(iii) Both previous effects are much more evident when increasing the value of the electromechanical coupling.

The obtained results are satisfying and show that the proposed control strategy has a beneficial effect on the stability of the system. Nevertheless, to evaluate the real performances of this controller, a further optimization of the parameters is required. This will be the object of forthcoming papers. 

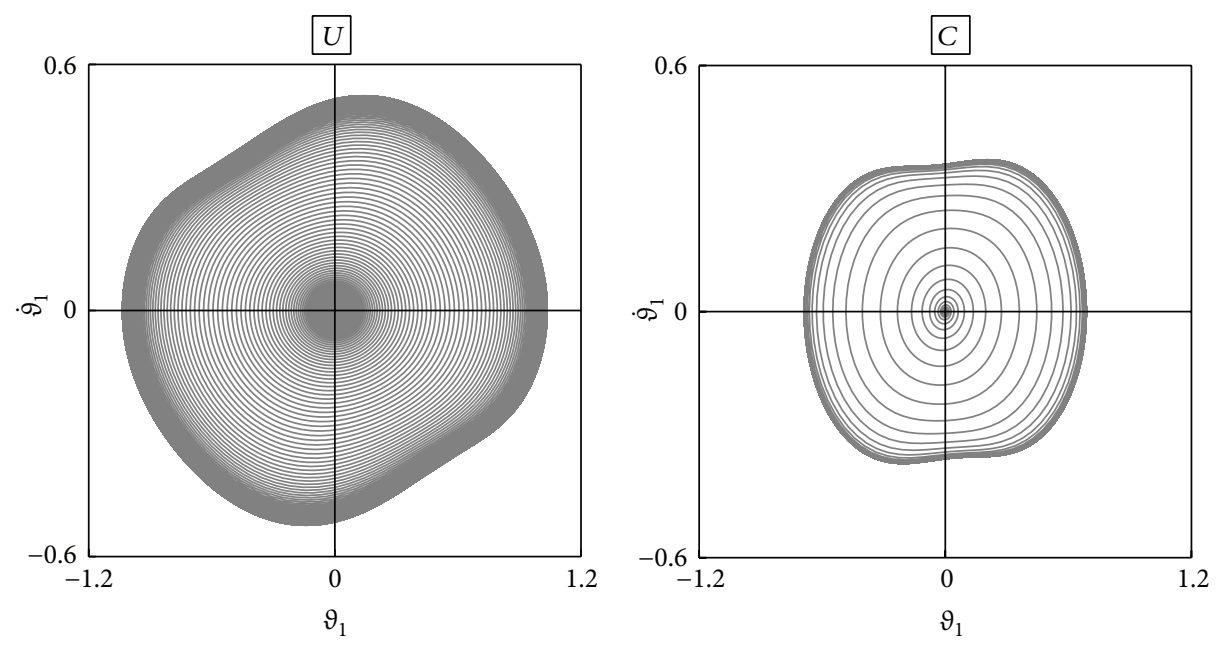

(a)
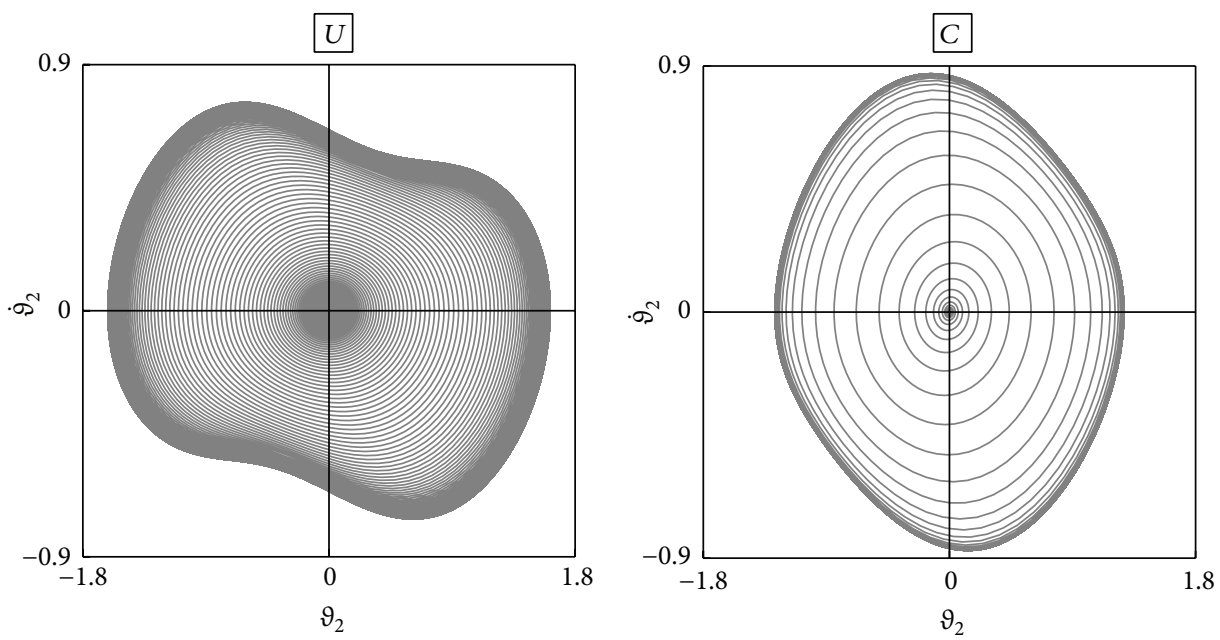

(b)
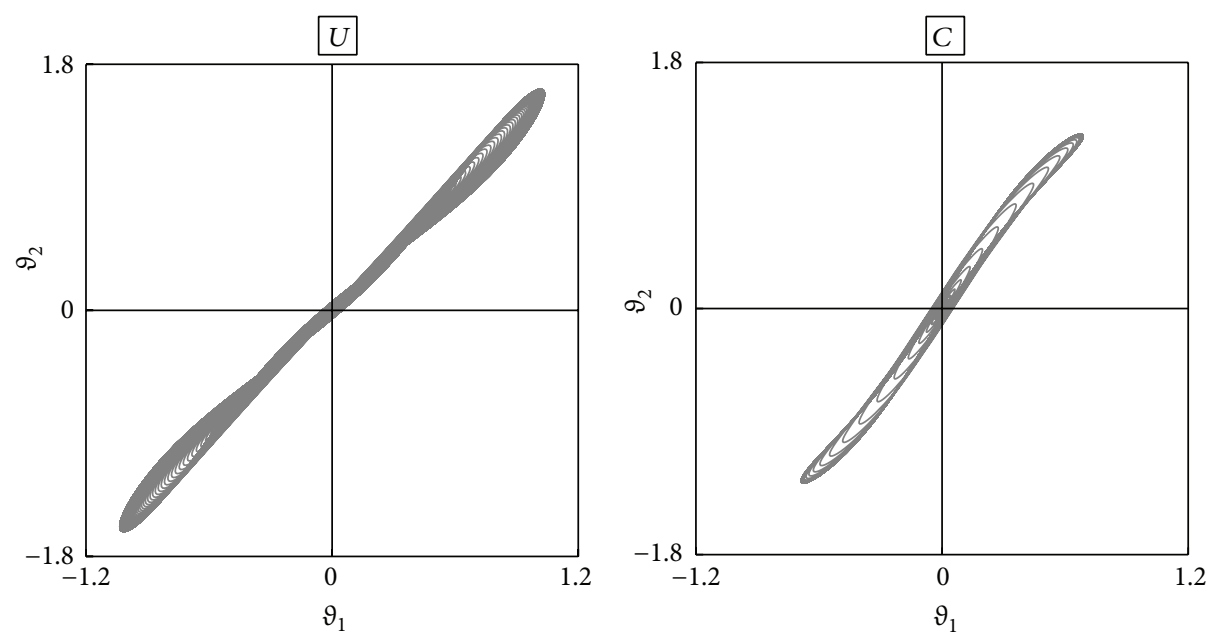

(c)

FIGURE 8: Numerical phase portraits for the case study I, when $\gamma=0.1$ and $\delta \mu=0.3$, for the uncontrolled (first column, $\mu \simeq 1.105$ ) and the controlled (second column, $\mu \simeq 1.88$ ) systems. 


\section{Conflict of Interests}

The authors declare that there is no conflict of interests regarding the publication of this paper.

\section{Acknowledgment}

This work was granted by the Italian Ministry of University and Research (MIUR), under the PRIN10-11 program, Project no. 2010MBJK5B.

\section{References}

[1] F. D’Annibale, G. Rosi, and A. Luongo, "On the failure of the 'Similar Piezoelectric Control' in preventing loss of stability by nonconservative positional forces," Zeitschrift für Angewandte Mathematik und Physik, vol. 66, no. 4, pp. 1949-1968, 2015.

[2] S. J. Elliott, P. Gardonio, T. C. Sors, and M. J. Brennan, "Active vibroacoustic control with multiple local feedback loops," The Journal of the Acoustical Society of America, vol. 111, no. 2, pp. 908-915, 2002.

[3] N. W. Hagood and A. von Flotow, "Damping of structural vibrations with piezoelectric materials and passive electrical networks," Journal of Sound and Vibration, vol. 146, no. 2, pp. 243-268, 1991.

[4] R. D. Blevins, Flow-Induced Vibration, Van Nostrand Reinhold, New York, NY, USA, 1990.

[5] M. Abe and Y. Fujino, "Dynamic characterization of multiple tuned mass dampers and some design formulas," Earthquake Engineering and Structural Dynamics, vol. 23, no. 8, pp. 813-835, 1994.

[6] R. Rana and T. T. Soong, "Parametric study and simplified design of tuned mass dampers," Engineering Structures, vol. 20, no. 3, pp. 193-204, 1998.

[7] V. Gattulli, F. Di Fabio, and A. Luongo, "Simple and double Hopf bifurcations in aeroelastic oscillators with tuned mass dampers," Journal of the Franklin Institute, vol. 338, no. 2-3, pp. 187-201, 2001.

[8] V. Gattulli, F. Di Fabio, and A. Luongo, "One to one resonant double Hopf bifurcation in aeroelastic oscillators with tuned mass dampers," Journal of Sound and Vibration, vol. 262, no. 2, pp. 201-217, 2003.

[9] U. Andreaus, L. Placidi, and G. Rega, "Microcantilever dynamics in tapping mode atomic force microscopy via higher eigenmodes analysis," Journal of Applied Physics, vol. 113, no. 22, Article ID 224302, 14 pages, 2013.

[10] F. D’Annibale, G. Rosi, and A. Luongo, "Linear stability of piezoelectric-controlled discrete mechanical systems under nonconservative positional forces," Meccanica, vol. 50, no. 3, pp. 825-839, 2015.

[11] F. D’Annibale, G. Rosi, and A. Luongo, "Piezoelectric control of Hopf bifurcations: a nonlinear discrete case study," International Journal of Non-Linear Mechanics, In press.

[12] A. F. Vakakis, L. A. Bergman, O. V. Gendelman et al., Nonlinear Targeted Energy Transfer in Mechanical and Structural Systems, vol. 156 of Solid Mechanics and Its Applications, Springer, Dordrecht, The Netherlands, 2009.

[13] A. Luongo and D. Zulli, "Dynamic analysis of externally excited NES-controlled systems via a mixed Multiple Scale/Harmonic Balance algorithm," Nonlinear Dynamics, vol. 70, no. 3, pp. 2049-2061, 2012.

[14] I. Giorgio, A. Culla, and D. Del Vescovo, "Multimode vibration control using several piezoelectric transducers shunted with a multiterminal network," Archive of Applied Mechanics, vol. 79, no. 9, pp. 859-879, 2009.
[15] G. Rosi, R. Paccapeli, F. Ollivier, and J. Pouget, "Optimization of piezoelectric patch positioning for passive sound radiation control of plates," Journal of Vibration and Control, vol. 19, no. 5, pp. $658-673,2013$

[16] S. Alessandroni, U. Andreaus, F. Dell'Isola, and M. Porfiri, "Piezo-ElectroMechanical (PEM) Kirchhoff-Love plates," European Journal of Mechanics, A/Solids, vol. 23, no. 4, pp. 689-702, 2004.

[17] C. Maurini, F. dell'Isola, and D. Del Vescovo, "Comparison of piezoelectronic networks acting as distributed vibration absorbers," Mechanical Systems and Signal Processing, vol. 18, no. 5, pp. 1243-1271, 2004.

[18] F. dell'Isola, C. Maurini, and M. Porfiri, "Passive damping of beam vibrations through distributed electric networks and piezoelectric transducers: prototype design and experimental validation," Smart Materials and Structures, vol. 13, no. 2, pp. 299-308, 2004.

[19] F. dell'Isola and S. Vidoli, "Continuum modelling of piezoelectromechanical truss beams: an application to vibration damping," Archive of Applied Mechanics, vol. 68, no. 1, pp. 1-19, 1998.

[20] F. Dell'Isola, E. G. Henneke, and M. Porfiri, "Synthesis of electrical networks interconnecting PZT actuators to damp mechanical vibrations," International Journal of Applied Electromagnetics and Mechanics, vol. 14, no. 1-4, pp. 417-424, 2002.

[21] F. Dell'Isola, F. Vestroni, and S. Vidoli, "A class of electromechanical systems: linear and nonlinear dynamics," International Journal of Applied Electromagnetics and Mechanics, vol. 40, no. 1, pp. 47-71, 2002.

[22] I. Giorgio, L. Galantucci, A. Della Corte, and D. Del Vescovo, "Piezo-electromechanical smart materials with distributed arrays of piezoelectric transducers: current and upcoming applications," International Journal of Applied Electromagnetics and Mechanics, vol. 47, no. 4, pp. 1051-1084, 2015.

[23] M. Porfiri, F. dell'Isola, and E. Santini, "Modeling and design of passive electric networks interconnecting piezoelectric transducers for distributed vibration control," International Journal of Applied Electromagnetics and Mechanics, vol. 21, no. 2, pp. 6987, 2005.

[24] S. Vidoli and F. dell'Isola, "Modal coupling in one-dimensional electromechanical structured continua," Acta Mechanica, vol. 141, no. 1-2, pp. 37-50, 2000.

[25] G. Rosi, J. Pouget, and F. Dell'Isola, "Control of sound radiation and transmission by a piezoelectric plate with an optimized resistive electrode," European Journal of Mechanics-A/Solids, vol. 29, no. 5, pp. 859-870, 2010.

[26] H. Ziegler, "Die Stabilitätskriterien der Elastomechanik," Ingenieur-Archiv, vol. 20, no. 1, pp. 49-56, 1952.

[27] O. N. Kirillov, "A theory of the destabilization paradox in nonconservative systems," Acta Mechanica, vol. 174, no. 3-4, pp. 145$166,2005$.

[28] H. Whitney, "The general type of singularity of a set of $2 n-1$ smooth functions of $n$ variables," Duke Mathematical Journal, vol. 10, no. 1, pp. 161-172, 1943.

[29] O. N. Kirillov and F. Verhulst, "Paradoxes of dissipationinduced destabilization or who opened Whitney's umbrella?" Zeitschrift für Angewandte Mathematik und Mechanik, vol. 90, no. 6, pp. 462-488, 2010 .

[30] A. Luongo and F. D'Annibale, "On the destabilizing effect of damping on discrete and continuous circulatory systems," Journal of Sound and Vibration, vol. 333, no. 24, pp. 6723-6741, 2014.

[31] J. Den Hartog, Mechanical Vibrations, McGraw-Hill, New York, NY, USA, 1956. 


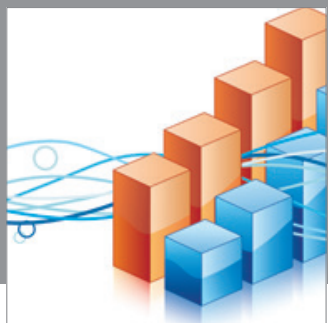

Advances in

Operations Research

mansans

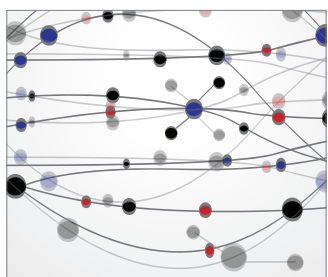

The Scientific World Journal
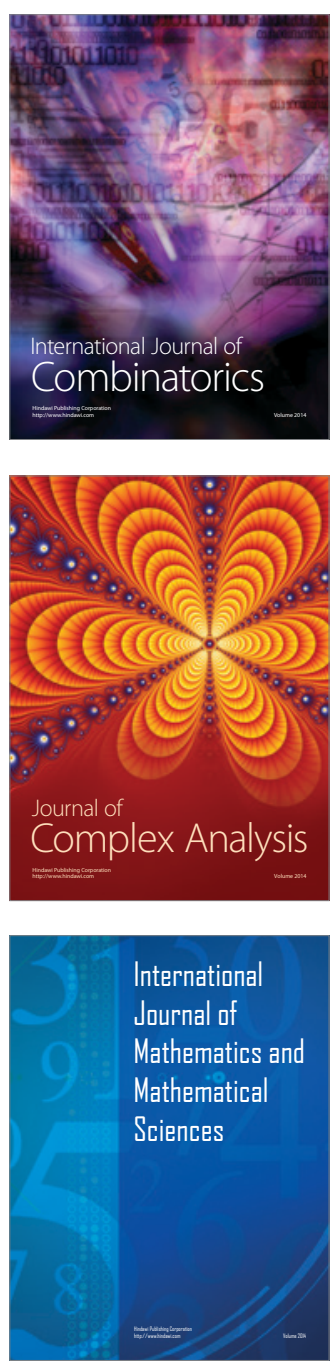
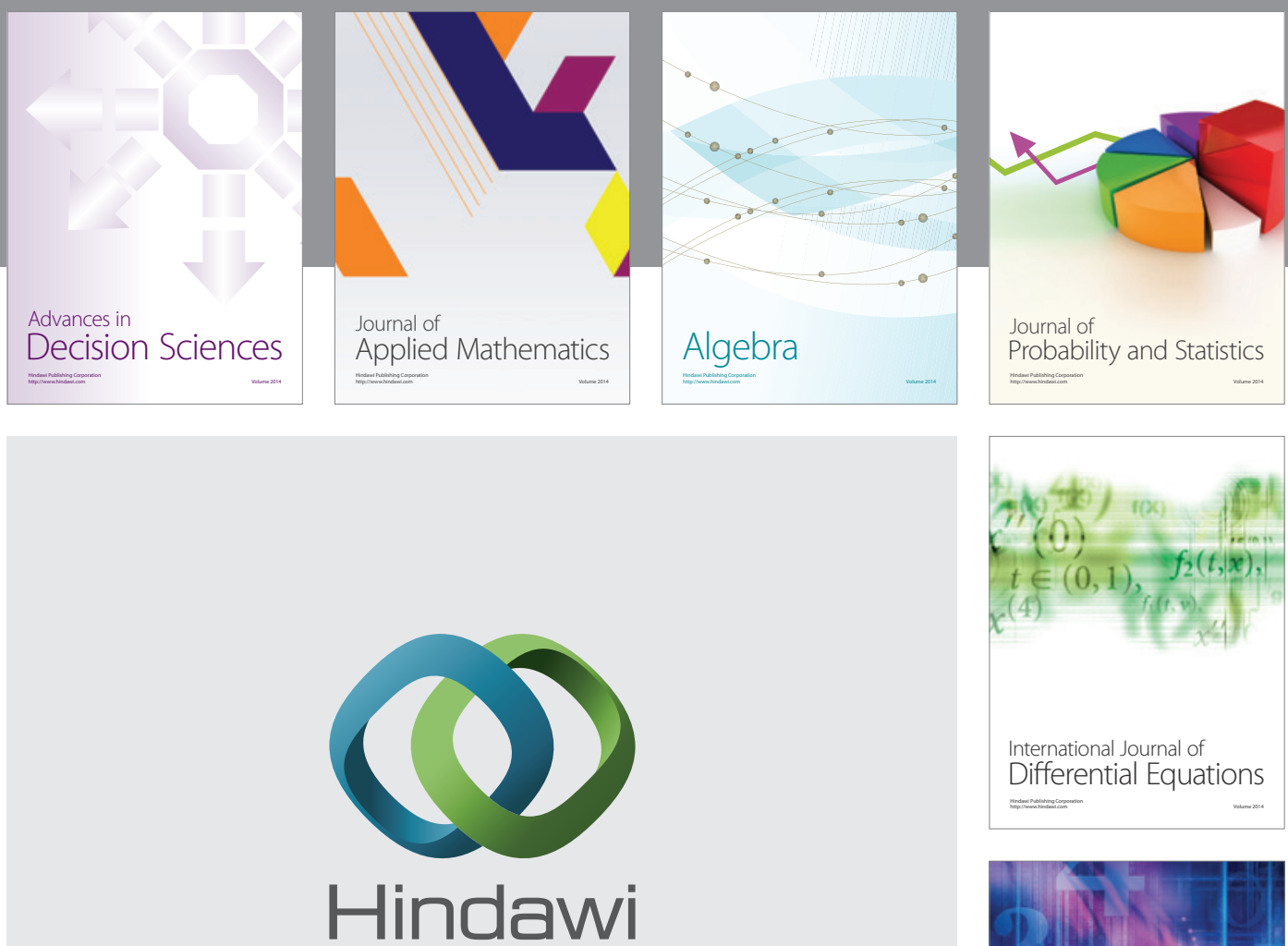

Submit your manuscripts at http://www.hindawi.com
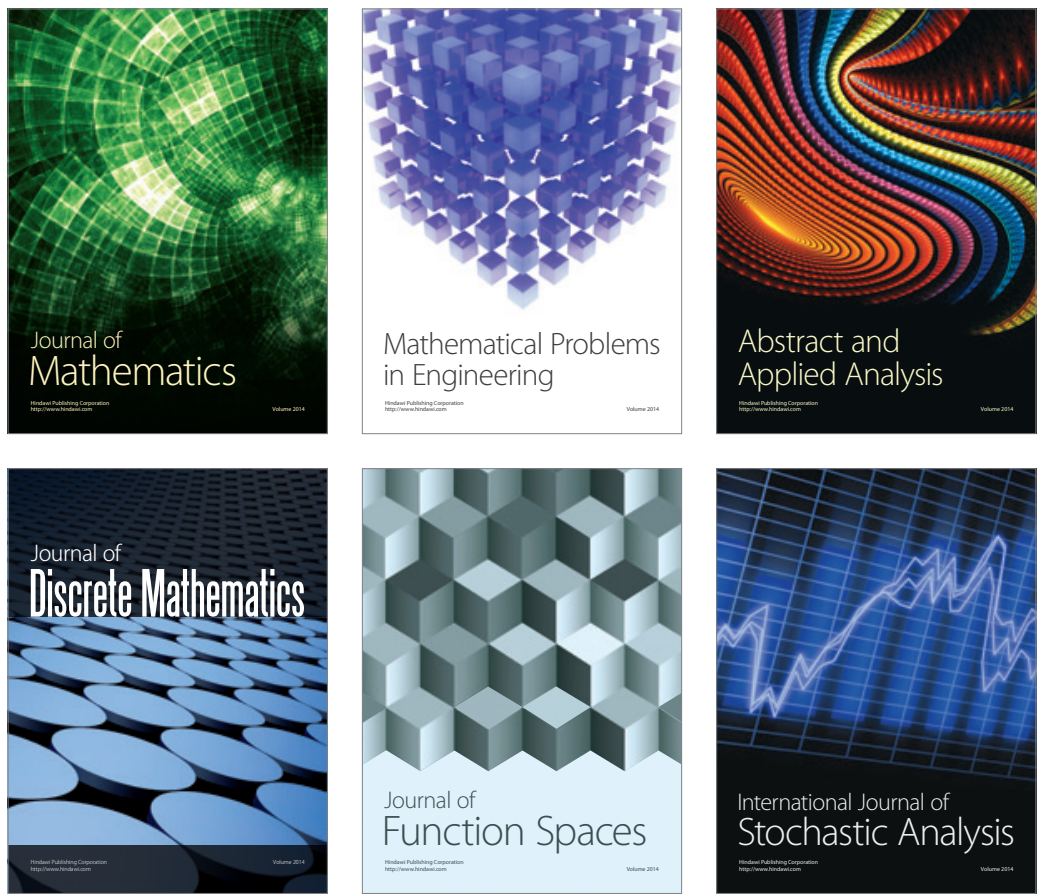

Journal of

Function Spaces

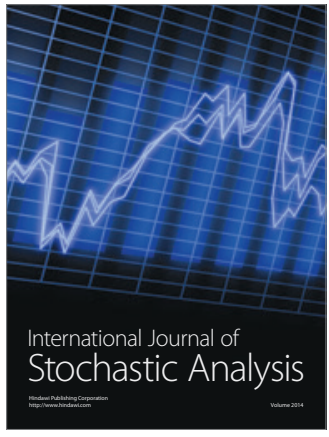

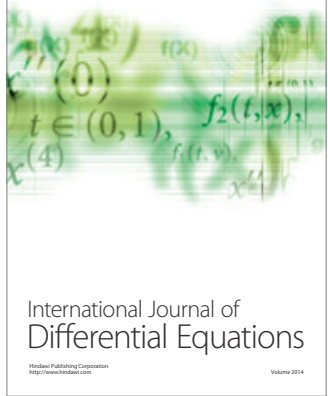
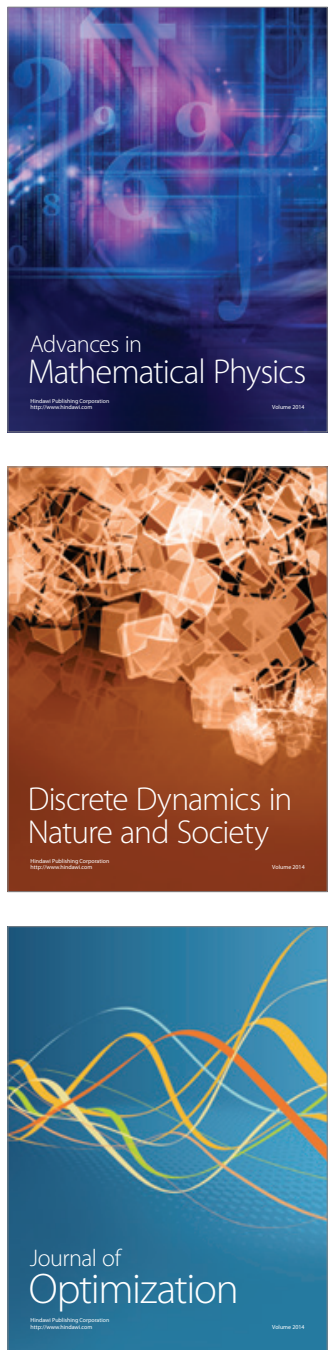